\title{
Symmetry of Spherically Converging Shock Waves through Reflection, Relating to the Shock Ignition Fusion Energy Scheme
}

\author{
C. J. Davie* and R. G. Evans \\ Imperial College London, London SW7 2AZ, United Kingdom
}

(Received 22 October 2012; published 30 April 2013)

\begin{abstract}
We examine the properties of perturbed spherically imploding shock waves in an ideal fluid through the collapse, bounce, and development into an outgoing shock wave. We find broad conservation of the size and shape of ingoing and outgoing perturbations when viewed at the same radius. The outgoing shock recovers the velocity of the unperturbed shock outside the strongly distorted core. The results are presented in the context of the robustness of the shock ignition approach to inertial fusion energy.
\end{abstract}

DOI: 10.1103/PhysRevLett.110.185002

PACS numbers: 52.57.Kk, 47.20.Ma, 47.40.Nm, 52.57.Fg

Shock ignition [1] is a variant on fast ignition [2] of inertial fusion targets which improves target performance by burning the deuterium-tritium fuel on a lower adiabat (and therefore with less input energy) than is possible using central ignition [1]. Shock ignition occurs as the result of the temperature increase at the surface of collision of two shock waves. The ingoing "ignition" shock is the result of a rapid late time increase in driver power and the outgoing shock is the earlier "compression" shock. This compression shock converges, reflects at its minimum radius, and then expands before colliding with the ignition shock. Full radiation hydrodynamics calculations [3] show that shock ignition is reasonably tolerant of departures from spherical symmetry. This is perhaps surprising since it is known [4,5] that spherically converging shock waves are unstable to perturbations of their surface.

The purpose of this Letter is to study an idealized problem relating to the compression shock, namely, the behavior of finite amplitude perturbations on a converging shock wave, to follow this shock through convergence, reflection at its minimum radius, and then into the expansion phase. We examine the underlying hydrodynamics with a uniform ideal gas, in the absence of heat conduction and other sources and sinks of energy. The relevance to shock ignition is that this approximates the behavior of the compression shock. Pure hydrodynamics is something of a worst case for these perturbed shocks-much of the additional physics will be dissipative and favor greater stability against perturbation growth. The results may also be of interest in the study of the symmetry of supernovae explosions with the proviso that the present work has no input of thermonuclear energy.

Previous work has established that during convergence the shock is unstable to perturbations on its surface [4,5], and that during the subsequent blast wave the perturbations are likely to decay [6]. We show that the perturbations are transferred with little change through convergence into expansion, recovering their approximate ingoing form. This ability of the spherical shock waves to recover their ingoing form is at the root of the robustness of shock ignition.

Analytic solutions for spherically symmetric converging shocks are used to validate the computer models for these shocks in 1D spherical and 2D axial symmetry. The 2D models then have small perturbations applied and are validated against linear solutions. This validated model is then applied to the nonlinear case including shock reflection.

The solution for an imploding, unperturbed spherical shock wave was derived by Guderley [7] and by Goldman [8] by means of a self-similar analysis of the basic continuity equations. The nature of the scale invariance of the self-similar solution means that it cannot be reproduced exactly in a finite system, but it is a good approximation on scale lengths between the system size and the molecular scale. The radius of the shock front $r$ as a function of time $t$ is given as

$$
r=\xi|t|^{\tilde{n}}
$$

When viewed at a fixed point in an Eulerian reference frame, the Guderley solution has three distinct phases. At times earlier than $t=-1$ the fluid is at rest, at $t=-1$ the passage of the ingoing shock causes the material to flow towards the center and its density increases due to the convergent flow. At $t=0$ the shock reflects at the origin, but its effect at the fixed observation point is not felt until, at $t>1.59$, the reflected shock wave causes a further compression of the fluid and a transition to an outwardly expanding flow.

Radius is normalized to this initial fixed point $r(t=-1)=1, \xi$ is the self-similarity coordinate and a different constant during convergence and reflection. For a monatomic gas Goldman [8] found that $\tilde{n}=0.688377$ and that density reaches the maximum, on the arrival of the reflected shock, of 32 times the initial density.

The stability of the ingoing shock front against small perturbations of its position was first investigated by Gardner, Book, and Bernstein [4] who showed that the perturbations were overstable; they oscillate with an increasing fractional distortion $\delta r / r$ although the absolute 
size $\delta r$ decreases with time. A more complete analysis of the growth of perturbations on a spherical shock front was given by K. Evans in the context of sonoluminescence [5].

These analytic treatments of perturbation growth serve to benchmark our work, but our aim is to follow the perturbed shock through the bounce or minimum radius and into its expanding phase. The lack of any expansion parameter through the vicinity of $r=0$ causes us to rely on computational methods and we are careful to verify the models at several stages of the work.

Since the scale invariant self-similar solution of Guderley cannot be transferred to a computational model with a finite grid, we construct an appropriate piston to drive the shock. We first make use of a result by Ribeyre et al. [9] who give an approximation to the velocity of a Lagrangian fluid element following the passage of the spherical shock wave. This is used as a boundary condition in a 1D spherical fluid model [10], set to model an ideal fluid with no dissipation apart from the shock itself. The results overlay the Guderley solution within the accuracy of plotting, shown in Fig. 1. We then use the same 1D spherical model to establish the behavior of the implosion of a finite thickness spherical shell of material which gives us an initial condition, the piston, that is easily transferred to other models. The inner surface of the spherical piston approximates the convergent shock and the thickness must be large enough that the rarefaction wave from the outer surface of the piston does not affect the shock dynamics until well after shock reflection.

Our 2D fluid model is the ZEUS2D [11] code, developed and widely used in the astrophysics community, set to follow the behavior of an ideal fluid. We use a cylindrical

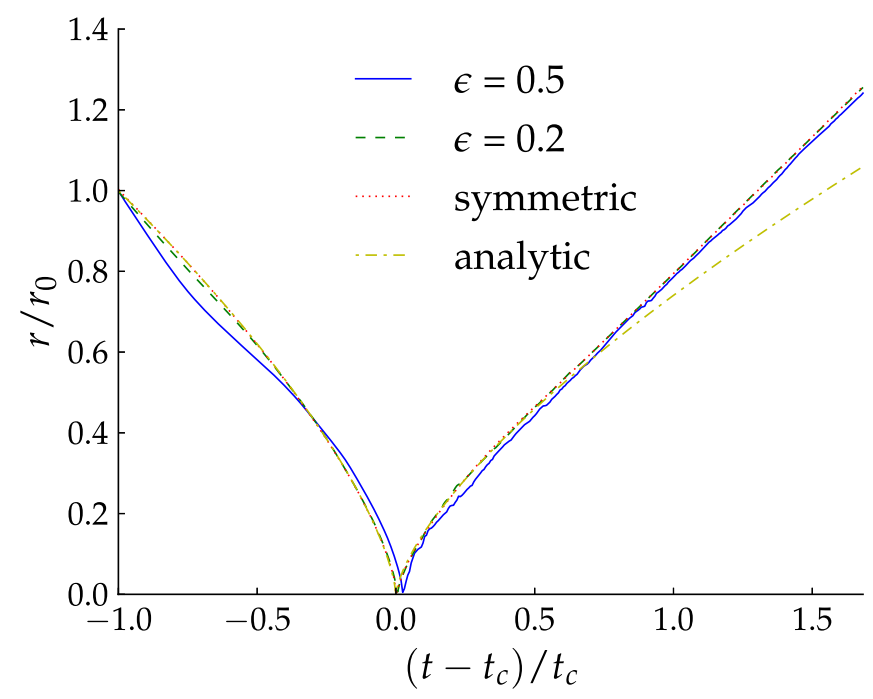

FIG. 1 (color online). The position of the shock front at a given time for the analytical solution and simulations with differing initial perturbation sizes for mode number $l=16$, using normalization corresponding to the Guderley solution. The symmetric simulation diverges from the analytic solution after moving into the rarefied material. $r-z$ grid to follow the implosion instead of an $r-\theta$ grid; the $r-\theta$ mesh gives rise to a very large interchange of the radial and angular components of velocity, as material passes close to the origin. It is a clear requirement that all results presented here are "grid converged" in the sense that our conclusions are unchanged if the resolution of the mesh is doubled.

At initialization, using a radial resolution ahead of the shock of typically 500 cells, we have a dynamic range of around 30 before the finite size of the grid limits our ability to measure the shock position.

ZEUS is initialized with the imploding piston of material described above, and in the spherically symmetric case (within the limitations of mapping onto a $r-z$ mesh) we reproduce the results of the equivalent 1D spherical model.

Perturbations of the shock front are then added to the 2D simulation. A least squares fit is calculated for the radial profile of material properties $f(r)$ in the 1D model and is then applied to ZEUS,

$$
f_{i}(r)=\sum_{n=0}^{N} a_{i, n} r^{n},
$$

where $i$ represents the material properties, the mass density $\rho$, velocity $v$, and energy density $e$. A quadric fit was found to be sufficiently good.

This transfer of evolved properties into an Eulerian solver leads to a spherical piston of material with two discontinuities. The inner discontinuity forms a shock front, while the outer discontinuity forms a rarefaction front. The shock solution remains approximately valid until these fronts meet.

The converging shock front is identified where the density increases to twice the background value. The reflected shock front is identified at the greatest density gradient.

Spatial perturbations are applied to the piston and the simulation is allowed to evolve. Perturbations are applied by modifying Eq. (2), the method for transferring the material properties, such that $r \rightarrow r_{l}(\theta)$,

$$
r_{l}(\theta)=r_{0}\left[1+\epsilon Y_{l}(\theta) / N\right],
$$

$\epsilon$ is an arbitrarily chosen size of perturbation. $Y_{l}(\theta) / N$ is the azimuthally symmetric spherical harmonic of mode number $l$, rescaled such that the poles are at \pm 1 .

Two methods for measuring the size of the perturbation are used. The first method tracks the peaks and troughs and finds the magnitude of the perturbation by subtracting the radius of an adjacent peak and trough. The second method involves decomposing the shock front position into its spherical harmonic coefficients, the spherical analogy of Fourier decomposition; the orthogonality of azimuthally symmetric spherical harmonics $Y_{l}(\theta)$ as a basis set allows $\alpha_{l^{\prime}}$, the coefficient that describes the size of mode $l^{\prime}$, to be found.

As the shock front converges, the average shock front radius and velocity are robust against a range of 


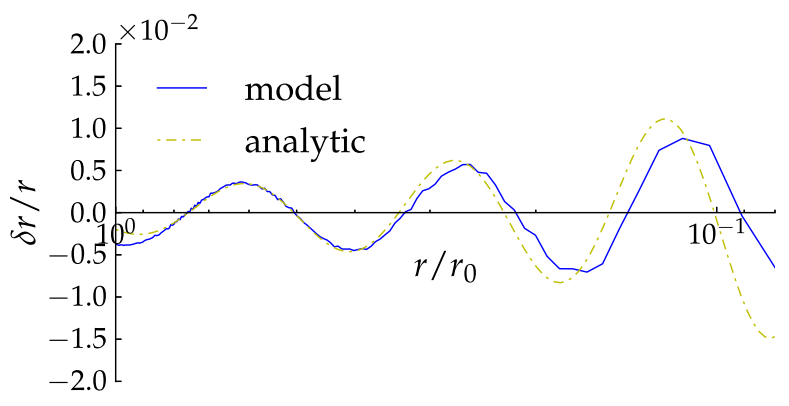

FIG. 2 (color online). Analytic and simulated perturbation size at a given shock front radius for an $l=16$ perturbation. The size of the perturbation relative to the shock radius $\delta r / r$ increases with decreasing radius $r$ for modes $l \geq 2$.

perturbation sizes, shown in Fig. 1. For these initial perturbation amplitudes the converging and reflected shock fronts closely track the symmetric simulation. The largest perturbations diverge from the symmetric solution but still recover as a broadly similar spherical shock.

Figure 2 shows the evolution of perturbed amplitudes in the simulations compared to the analytic solution found by K. Evans [5]. The computed oscillatory behavior and growth initially match the analytic solution well, eventually diverging, due to two effects: first, the analytic theory is valid only for "small" perturbations and, second, the perturbation initialized via Eqs. (2) and (3) does not precisely match a single radial eigenmode.

A number of cases are considered: small perturbation growth for high and lower mode perturbations, multiple modes, and large perturbations.

Figure 3 shows the growth of perturbations with different initial amplitudes during the convergence of the shock front. For mode $l=16$, perturbation amplitude saturates at $\delta r / r=(3.5 \pm 0.2) \times 10^{-2}$, perturbations with initial amplitudes smaller than this grow as expected, but perturbations with initial amplitudes greater than this diminish in time. Higher $l$ modes saturate at smaller amplitudes,
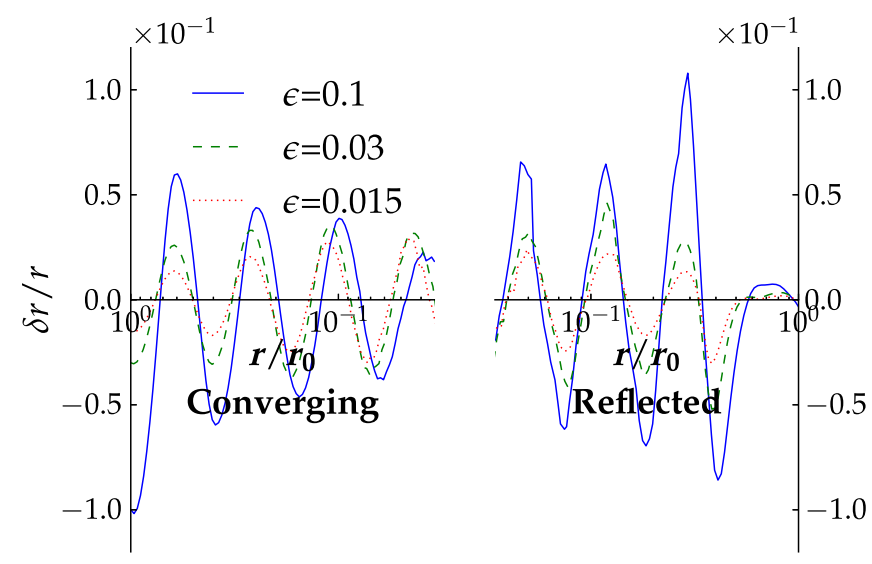

FIG. 3 (color online). Perturbation growth with average shock front radii for different initial $l=16$ perturbation amplitudes $\epsilon$ from Eq. (3), for both the converging and the reflected shock.

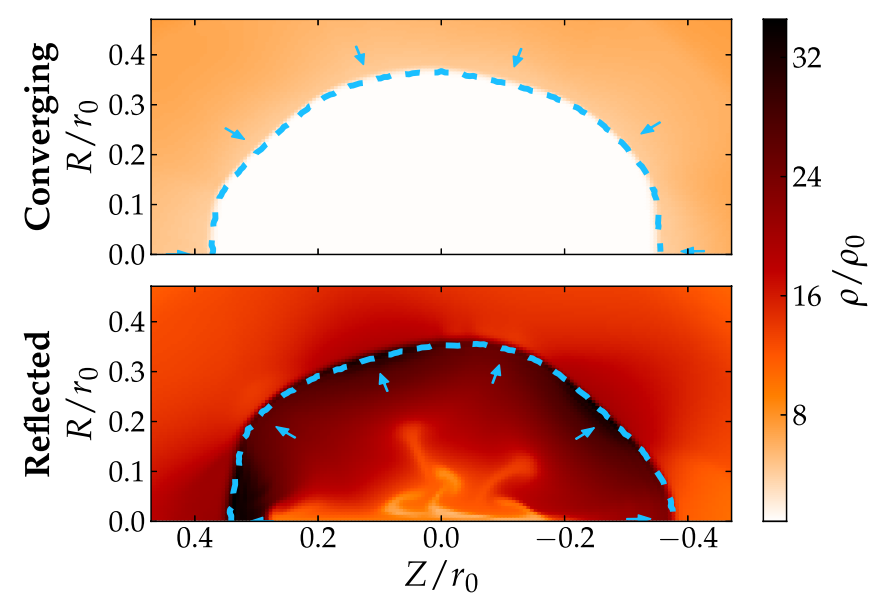

FIG. 4 (color online). Density profile of the converging and reflected shock at the same shock front radius. The dashed lines show the shock front position while the arrows show the direction of movement.

$l=32$ saturates at $\delta r / r=(7 \pm 1.5) \times 10^{-3}$ and $l=8$ saturates at $\delta r / r=(1.1 \pm 0.3) \times 10^{-1}$.

The saturated amplitude scales approximately as $l^{-2}$, which suggests that its origin is related to the change in sign of the curvature of the shock front as its amplitude increases. When $\delta r / r=1 / l^{2}$ the inward perturbation becomes concave rather than convex, we expect it to stop growing by comparison with the stability of planar shock waves, with convex outward and concave inward perturbations. The observed saturation amplitude is a few times larger than this simple calculation probably due to azimuthally symmetric perturbations only changing one of the two radii of curvature and also because the perturbation may continue to grow during the other half cycle when its curvature is again convex.

Figure 4 shows a density plot of two shock fronts, initialized with a mode $l=5$ perturbation, at the same radius during convergence and subsequent expansion. Perturbation size and shape are similar before and after

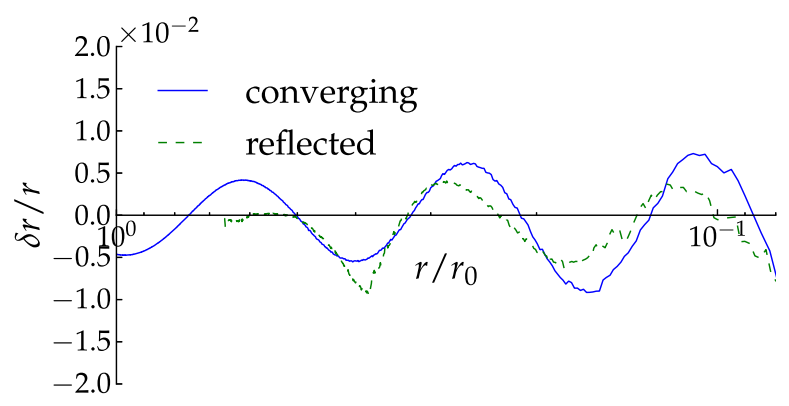

FIG. 5 (color online). Amplitude of the perturbation for converging and reflected shock fronts, with initial perturbation mode number $l=16$. As the reflected shock front expands, it moves through more rarefied material and diverges from the behavior of the converging shock. 


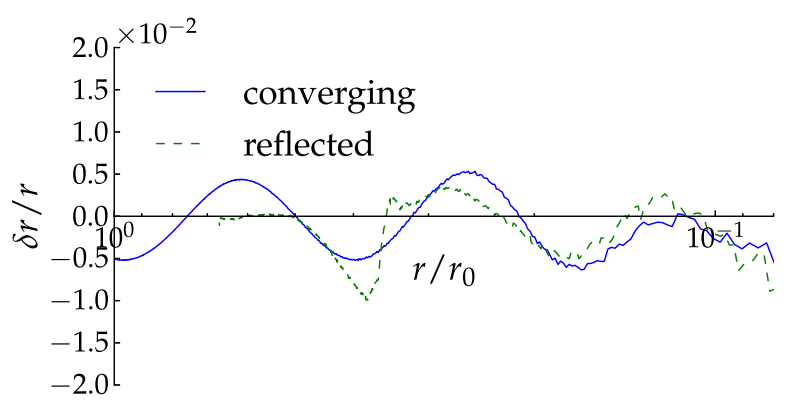

FIG. 6 (color online). $l=16$ perturbation growth found through decomposition into spherical harmonics for a multimode $l=16$ and $l^{\prime}=4$ initialization.

reflection; the mode behavior is clearly preserved, as is the underlying spherical shock front.

Perturbation peaks remain close to their initialized angular position throughout convergence and reflection, but these peaks oscillate, in general, at different radii during convergence and after reflection.

The recovery of shock front shape after reflection is our most important result and is shown more quantitatively in Fig. 5. Time advances left to right during convergence and right to left during expansion. This conservation of size and shape of perturbations is somewhat explained by the selflimiting behavior of perturbations.

After reflection, it is expected that a spherically expanding shock stabilizes-expanding shock fronts in static fluids are described by Sedov [12] and Taylor [13] and have been shown to be stable [6].

Since the fluid equations are nonlinear, multiple harmonic mode perturbations will couple together and typically seed the growth of other modes. As an example, we initialize the shock front to have the radial position perturbations with contributions from two modes, modifying Eq. (3), $r_{l, l^{\prime}}(\theta)=r_{0}\left\{1+\epsilon\left[Y_{l}(\theta) / N+Y_{l^{\prime}}(\theta) / N\right]\right\}$.

Comparing this two-mode case, Fig. 6 , and the singlemode case, Fig. 5, for much of the convergence there is very little mode-mode coupling between modes $l=4$ and $l=16$. As in the single-mode case, the shape and size of the perturbation is again preserved through reflection, although near convergence other seeded modes become more prominent.

These spatial perturbations on shock fronts correspond to certain types of variation from symmetry, for example, due to surface roughness of an inertial fusion target. Similarly, pressure perturbations behind the shock front correspond to different variations from symmetry, for example, variations in the power deposited on the target from the mechanism driving the implosion. Converging shock waves driven by nonuniform pressure show additional effects such as the converging jets depicted in Ref. [4], and large pressure perturbations can clearly induce extreme behavior. For perturbations in drive pressure less than $10 \%$ or so, our main conclusions remain valid; namely, outside of a small core region the shock velocity after the bounce recovers the value of the symmetric case, and perturbation amplitude before and after reflection are closely similar when measured at the same radius. Further analysis of this significant additional effect goes beyond the scope of this Letter.

Our results show that the inherent robustness of shock ignition against modest departures from spherical symmetry is based in the underlying robustness of the process of shock reflection. Since the ideal fluid equations preserve energy and momentum and Taylor-Sedov expansions are stable, it is intuitively clear why the expanding shock wave recovers the velocity of the symmetric case. The approximate recovery of shock front shape after reflection is perhaps more surprising and may relate to the existence of higher moments of the fluid motion which are similarly conserved.

We thank AWE for their financial assistance in support of the doctoral research of C. J. D.

*c.davie10@imperial.ac.uk

[1] R. Betti, C. D. Zhou, K.S. Anderson, J. L. Perkins, W. Theobald, and A. A. Solodov, Phys. Rev. Lett. 98, 155001 (2007).

[2] M. Tabak, J. Hammer, M. E. Glinsky, W. L. Kruer, S. C. Wilks, J. Woodworth, E. M. Campbell, M. D. Perry, and R. J. Mason, Phys. Plasmas 1, 1626 (1994).

[3] S. Atzeni, A. Schiavi, and A. Marocchino, Plasma Phys. Controlled Fusion 53, 035010 (2011).

[4] J.H. Gardner, D. L. Book, and I. B. Bernstein, J. Fluid Mech. 114, 41 (2006).

[5] A. K. Evans, Phys. Rev. E 54, 5004 (1996).

[6] E. T. Vishniac and D. Ryu, Astrophys. J. 337, 917 (1989).

[7] G. Guderley, Luftfahrtforschung 19, 302 (1942).

[8] E. B. Goldman, Plasma Phys. 15, 289 (1973).

[9] X. Ribeyre, V. T. Tikhonchuk, J. Breil, M. Lafon, and E. Le Bel, Phys. Plasmas 18, 102702 (2011).

[10] J.P. Christiansen, D.E.T.F. Ashby, and K. V. Roberts, Comput. Phys. Commun. 7, 271 (1974).

[11] J. M. Stone and M. L. Norman, Astrophys. J. Suppl. Ser. 80, 753 (1992).

[12] L. I. Sedov, J. Appl. Math. Mech. 10, 241 (1946).

[13] G. Taylor, Proc. R. Soc. A 201, 192 (1950). 